\title{
Comparison of the identification of coagulase-negative staphylococci by matrix-assisted laser desorption ionization time-of-flight mass spectrometry and tuf sequencing
}

\author{
N. Carpaij • R. J. L. Willems • M. J. M. Bonten • \\ A. C. Fluit
}

Received: 3 February 2011 /Accepted: 11 February 2011 /Published online: 26 February 2011

(C) The Author(s) 2011. This article is published with open access at Springerlink.com

\begin{abstract}
The increasing incidence of coagulase-negative staphylococci (CoNS) in hospital-acquired infections underlines the need for an accurate and simple identification of Staphylococcus isolates at the species level. Sequencing of the tuf gene has been shown to be the most accurate for the species identification of CoNS. We determined the species of 62 consecutive clinical and 31 reference CoNS isolates by tuf gene sequencing and matrixassisted laser desorption ionization time-of-flight mass spectrometry (MALDI-TOF-MS). Species assignment by MALDI-TOF-MS and tuf sequencing was congruent in all cases. We conclude that MALDI-TOF-MS is accurate for identifying CoNS in routine clinical practice. The study also identified an unexpectedly high number of cases of Staphylococcus capitis infections among 62 consecutive CoNS isolates in 2009 at the University Medical Center Utrecht, the Netherlands.
\end{abstract}

\section{Introduction}

Coagulase-negative staphylococci (CoNS) maintain a benign relationship with their host and are only considered to be

\footnotetext{
N. Carpaij • R. J. L. Willems • M. J. M. Bonten - A. C. Fluit Department of Medical Microbiology,

University Medical Center Utrecht, room G04.614,

P.O. Box 85500, 3508 GA Utrecht, The Netherlands

\section{N. Carpaij $(\bowtie)$}

Department of Medical Microbiology,

University Medical Center Utrecht, room G04.643,

Heidelberglaan 100,

3485 CX Utrecht, The Netherlands

e-mail: N.Carpaij-2@umcutrecht.nl
}

pathogenic when natural barriers are damaged, usually due to trauma or the implantation of medical devices [1,2]. Rates of CoNS infections in hospitals have increased, which necessitates the need for the accurate and simple identification of Staphylococcus isolates at the species level [3-5].

CoNS that are commonly isolated from humans are Staphylococcus epidermidis, Staphylococcus capitis, Staphylococcus hominis, Staphylococcus haemolyticus, Staphylococcus warneri, Staphylococcus caprae, Staphylococcus saccharolyticus, Staphylococcus pasteuri, Staphylococcus saprophyticus, and Staphylococcus lugdunensis [4]. It is notheworthy that some species (i.e., S. epidermidis, S. haemolyticus, S. lugdunensis, S. warneri, and Staphylococcus schleiferi) are more often found in severe human infections, such as endocarditis $[4,6]$. Molecular methods targeting the 16S rRNA, hsp60, femA, $r p o B$, gap, tuf, and $\operatorname{sod} A$ genes are currently favored for accurate identification, but these methods are timeconsuming and expensive $[5,7]$.

Here, we evaluated the use of matrix-assisted laser desorption ionization time-of-flight mass spectrometry (MALDI-TOF-MS) (Bruker Daltonics, Bremen, Germany) for the species determination of 62 consecutive clinical CoNS isolates causing nosocomial infections at the University Medical Center Utrecht (UMCU), the Netherlands. As the gold standard for CoNS species determination, we used tuf gene sequencing, because this gene was shown to be the most accurate for specifying CoNS $[7,8]$.

\section{Materials and methods}

In total, 62 clinical CoNS were included, as were 31 reference isolates, representing S. capitis, Staphylococcus 
sciuri, S. epidermidis, S. saprophyticus, Staphylococcus xylosus, S. hominis, S. haemolyticus, and S. warneri, obtained from either the ATCC or a previous study [7].

The 62 clinical CoNS isolates originated from hospitalized patients with invasive infections and were collected in the first two months of 2009. The isolates were classified as CoNS on the basis of the morphology and a fast agglutination test (Remel, Badhoevedorp, the Netherlands).

For tuf sequencing, genomic DNA of the isolates was used for amplifying and sequencing a 412-bp fragment of the tuf gene with the primers tuf-F and tuf-R [7]. The tuf gene sequences were aligned and a neighbor-joining tree was constructed using MEGA 4.1 with the maximum composite likelihood model assuming rate uniformity and pattern homogeneity [9].

For all isolates, the tuf gene was sequenced with species determination based on alignments with reference isolates. All but two isolates $(98 \%)$ could be assigned to the species level using a cut-off of $98 \%$ similarity (Table 1 and Fig. 1 ). BLAST searches of the two non-assigned isolates revealed that one isolate had a $t u f$ sequence $100 \%$ identical to the $t u f$ gene of Staphylococcus simulans ATCC27848, and the tuf gene of the other was $99 \%$ similar to that of $S$. lugdunensis HKU 09-01.

For the MALDI-TOF-MS analysis, material from a single colony of a fresh overnight culture was placed onto a steel target plate and processed as described previously [10]. Raw spectra were analyzed by MALDI Biotyper 2.0 software (Bruker Daltonics) with default settings. An internal control (Escherichia coli DH5 $\alpha$ ) was used for calibration before each experiment. Identification scores above 2 or between 1.8 and 2 for duplicate samples were considered to be reliable [10].

\section{Results and discussion}

All reference and clinical isolates yielded reliable identification by MALDI-TOF-MS. Species assignment by MALDI-TOF-MS and tuf sequencing was congruent in all cases, implying an accuracy of species identification by MALDI-TOF-MS of $100 \%$.

The majority of the clinical isolates were classified as S. epidermidis $(n=33,53 \%)$ and S. haemolyticus $(n=13$, $20 \%$ ) (Table 1). Both CoNS species are often associated with infections in hospitalized patients [4]. Surprisingly, ten of 62 isolates (16\%) from 2009 were classified as S. capitis, a species normally rarely encountered as an etiological agent of nosocomial infections. These ten isolates were recovered from ten different patients, of which nine appeared in two epidemiologically distinct clusters of five
Table 1 Species identification of coagulase-negative staphylococci (CoNS) by tuf and matrix-assisted laser desorption ionization time-of-flight mass spectrometry (MALDI-TOF-MS)

\begin{tabular}{|c|c|c|}
\hline & S. epidermidis $(n=5$ & \\
\hline & Reference isolates & 17 \\
\hline & Clinical isolates & $33(53 \%)$ \\
\hline & S. haemolyticus $(n=$ & \\
\hline & Reference isolates & 2 \\
\hline & Clinical isolates & $12(19 \%)$ \\
\hline & S. hominis $(n=6)$ & \\
\hline & Reference isolates & 2 \\
\hline & Clinical isolates & $4(6 \%)$ \\
\hline & S. lugdunensis $(n=1$ & \\
\hline & Reference isolates & 0 \\
\hline & Clinical isolates & $1(2 \%)$ \\
\hline & S. saprophyticus ( $n=$ & \\
\hline & Reference isolates & 1 \\
\hline & Clinical isolates & 0 \\
\hline & S. sciuri $(n=1)$ & \\
\hline & Reference isolates & 1 \\
\hline & Clinical isolates & 0 \\
\hline & S. simulans $(n=1)$ & \\
\hline & Reference isolates & 0 \\
\hline & Clinical isolates & $1(2 \%)$ \\
\hline & S. warneri $(n=2)$ & \\
\hline & Reference isolates & 1 \\
\hline & Clinical isolates & $1(2 \%)$ \\
\hline & S. xylosus $(n=4)$ & \\
\hline & Reference isolates & 4 \\
\hline & Clinical isolates & 0 \\
\hline
\end{tabular}

and four patients (stayed on the same ward during overlapping time periods), suggesting multiple events of cross-transmission (data not shown).

In this study, we demonstrated that MALDI-TOF-MS, compared to tuf sequencing, is an accurate method for the species identification of routinely isolated clinically important CoNS. The usefulness of MALDI-TOF-MS for the species determination of CoNS has been reported previously, but MALDI-TOF-MS was not compared to tuf sequence-based determination in these studies $[5,6,10$, 11]. However, Bergeron et al. concluded in their set of isolates, which also included isolates that are not found in a clinical setting, that tuf was better for species determination than MALDI-TOF-MS [8]. Because MALDI-TOF-MS is easy to perform, fast, and relatively cheap, it is the preferred method for the species identification of clinical CoNS in routine clinical microbiology. 
Fig. 1 Neighbor-joining tree based on the tuf gene sequences of 93 Staphylococcus isolates included in this study. The reference isolates are indicated with the species name and the correlating ATCC number or the number used in the study of Heikens et al. [7]. The clinical isolates are designated CoNS 1 to CoNS 62. The two isolates that did not align with a reference isolate and for which the species was identified using BLAST analysis are indicated as S. lugdunensis - CoNS 44 and S. simulans - CoNS 6. The different colors indicate the different species: pink lineages indicate $S$. epidermidis isolates, dark blue indicates $S$. warneri, light blue indicates $S$. capitis, green indicates $S$. hominis, yellow indicates

S. haemolyticus, and red indicates $S$. xylosus

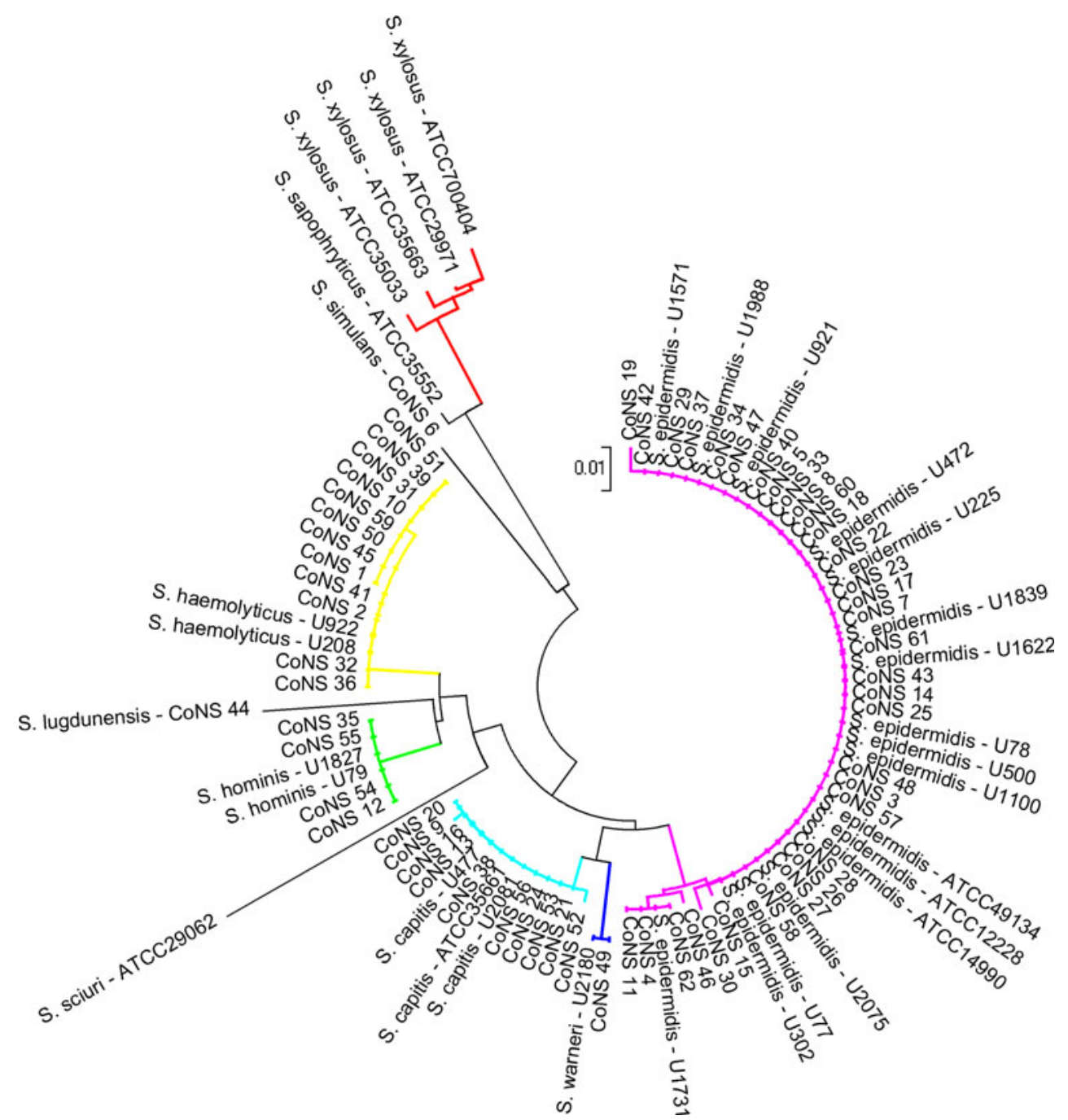

Acknowledgments M.J.M.B. was supported by the Netherlands Organisation for Scientific Research (VICI NWO Grant 918.76.611).

Open Access This article is distributed under the terms of the Creative Commons Attribution Noncommercial License which permits any noncommercial use, distribution, and reproduction in any medium, provided the original author(s) and source are credited.

\section{References}

1. Otto M (2009) Staphylococcus epidermidis - the 'accidental' pathogen. Nat Rev Microbiol 7:555-567

2. Paradisi F, Corti G, Messeri D (2001) Antistaphylococcal (MSSA, MRSA, MSSE, MRSE) antibiotics. Med Clin North Am 85:117

3. Hidron AI, Edwards JR, Patel J, Horan TC, Sievert DM, Pollock DA, Fridkin SK (2008) NHSN annual update: antimicrobial-resistant pathogens associated with healthcareassociated infections: annual summary of data reported to the
National Healthcare Safety Network at the Centers for Disease Control and Prevention, 2006-2007. Infect Control Hosp Epidemiol 29:996-1011

4. Huebner J, Goldmann DA (1999) Coagulase-negative staphylococci: role as pathogens. Annu Rev Med 50:223-236

5. Spanu T, De Carolis E, Fiori B, Sanguinetti M, D’Inzeo T, Fadda G, Posteraro B (2011) Evaluation of matrix-assisted laser desorption ionization-time-of-flight mass spectrometry in comparison to $r р о B$ gene sequencing for species identification of bloodstream infection staphylococcal isolates. Clin Microbiol Infect 17:44-49

6. Dupont C, Sivadon-Tardy V, Bille E, Dauphin B, Beretti JL, Alvarez AS, Degand N, Ferroni A, Rottman M, Herrmann JL, Nassif X, Ronco E, Carbonnelle E (2010) Identification of clinical coagulase-negative staphylococci, isolated in microbiology laboratories, by matrix-assisted laser desorption/ionization-time of flight mass spectrometry and two automated systems. Clin Microbiol Infect 16:998-1004

7. Heikens E, Fleer A, Paauw A, Florijn A, Fluit AC (2005) Comparison of genotypic and phenotypic methods for specieslevel identification of clinical isolates of coagulase-negative staphylococci. J Clin Microbiol 43:2286-2290 
8. Bergeron M, Dauwalder O, Gouy M, Freydiere AM, Bes M, Meugnier H, Benito Y,Etienne J, Lina G, Vandenesch F, Boisset S (2011) Species identification ofstaphylococci by amplification and sequencing of the tuf gene compared to the gap geneand by matrix-assisted laser desorption ionization time-of-flight mass spectrometry. Eur J Clin Microbiol Infect Dis 30:343-354

9. Tamura K, Dudley J, Nei M, Kumar S (2007) MEGA4: Molecular Evolutionary Genetics Analysis (MEGA) software version 4.0. Mol Biol Evol 24:1596-1599
10. Cherkaoui A, Hibbs J, Emonet S, Tangomo M, Girard M, Francois P, Schrenzel J (2010) Comparison of two matrix-assisted laser desorption ionization-time of flight mass spectrometry methods with conventional phenotypic identification for routine identification of bacteria to the species level. J Clin Microbiol 48:1169-1175

11. Bessède E, Angla-Gre M, Delagarde Y, Sep Hieng S, Ménard A, Mégraud F (2010) Matrix-assisted laser-desorption/ionization biotyper: experience in the routine of a University hospital. Clin Microbiol Infect (in press) 